\title{
DISCOVERY OF AN X-RAY JET AND EXTENDED JET STRUCTURE IN THE QUASAR PKS 1055+201
}

\author{
D. A. Schwartz, ${ }^{1}$ H. L. Marshall, ${ }^{2}$ J. E. J. Lovell,${ }^{3}$ D. W. Murphy, ${ }^{4}$ G. V. Bicknell, ${ }^{5}$ M. Birkinshaw, ${ }^{1,6}$ \\ J. M. Gelbord, ${ }^{2}$ M. Georganopoulos, ${ }^{7,8}$ L. Godfrey, ${ }^{3,5}$ D. L. Jauncey, ${ }^{3}$ S. Jester, ${ }^{9}$ E. S. Perlman, ${ }^{7}$ and D. M. Worrall ${ }^{1,6}$ \\ Received 2006 June 23; accepted 2006 July 10; published 2006 August 7
}

\begin{abstract}
This Letter reports rich X-ray jet structures found in the Chandra observation of PKS $1055+201$. In addition to an X-ray jet coincident with the radio jet, we detect a region of extended X-ray emission surrounding the jet as far from the core as the radio hot spot to the north, and a similar extended X-ray region along the presumed path of the unseen counterjet to the southern radio lobe. Both X-ray regions show a similar curvature to the west, relative to the quasar. We interpret this as the first example in which we separately detect the X-ray emission from a narrow jet and extended, residual jet plasma over the entire length of a powerful FR II jet.
\end{abstract}

Subject headings: galaxies: jets — quasars: general — quasars: individual (PKS 1055+201, 4C 20.24) — X-rays: galaxies

\section{INTRODUCTION}

The surprising discovery of a luminous, $100 \mathrm{kpc}$ scale $\mathrm{X}$ ray jet in the initial observations of PKS 0637-752 (Schwartz et al. 2000; Chartas et al. 2000) demonstrated that Chandra had opened a new channel of data for the study of extragalactic jets. One of the extreme cases discovered in our survey for Xray emission from radio-bright quasar jets (Marshall et al. 2005; Gelbord et al. 2005) is the FR II radio source PKS 1055+201 (Shimmins \& Day 1968). Shimmins \& Day (1968) associated it with the previously discovered 4C 20.24 (Pilkington \& Scott 1965). Bolton et al. (1968) identified the object as a quasar at redshift $z=1.11$. We proposed Chandra follow-up observations based on our initial $4.7 \mathrm{ks}$ image (obs/04889), which showed that the X-ray jet followed the $21^{\prime \prime}$ length of the radio jet. At the redshift of the quasar, $1^{\prime \prime}=8.24 \mathrm{kpc}$ in the plane of the sky. ${ }^{10}$ Since we infer that the jet is in relativistic motion with Doppler factor $\delta \approx 6$ (Schwartz et al. 2006b), the jet must be at an angle no more than $9^{\circ}$ from our line of sight, so that its total deprojected length is of order $1 \mathrm{Mpc}$.

In this Letter we discuss the extended X-ray emission surrounding the prominent radio and X-ray jet and the inferred, invisible counterjet. The ability to observe both the jet and $\mathrm{X}$ -

\footnotetext{
${ }^{1}$ Harvard-Smithsonian Center for Astrophysics, 60 Garden Street, Cambridge, MA 02138; das@head-cfa.harvard.edu.

${ }^{2}$ Kavli Institute for Astrophysics and Space Research, Massachusetts Institute of Technology, 77 Massachusetts Avenue, Cambridge, MA 02139; hermanm@space.mit.edu, jonathan@space.mit.edu.

${ }^{3}$ CSIRO Australia Telescope National Facility, P.O. Box 76, Epping, NSW 1710, Australia; jim.lovell@ csiro.au, djauncey@atnf.csiro.au.

${ }^{4}$ Jet Propulsion Laboratory, 4800 Oak Grove Drive, Pasadena, CA 91109; dwm@sgra.jpl.nasa.gov.

${ }^{5}$ Research School of Astronomy and Astrophysics, Australian National University, Cotter Road, Weston Creek, Canberra, ACT 72611, Australia; geoff@ mso.anu.edu.au, lgodfrey@mso.anu.edu.au.

${ }^{6}$ Department of Physics, University of Bristol, Tyndall Avenue, Bristol BS8 1TL, UK; mark.birkinshaw@bristol.ac.uk, d.worrall@bristol.ac.uk.

${ }^{7}$ Department of Physics, Joint Center for Astrophysics, University of Maryland-Baltimore County, 1000 Hilltop Circle, Baltimore, MD 21250; markos@ jca.umbc.edu,perlman@jca.umbc.edu.

${ }^{8}$ NASA's Goddard Space Flight Center, Mail Code 660, Greenbelt, MD 20771.

${ }^{9}$ School of Physics and Astronomy, University of Southampton, Southampton SO17 1BJ, UK; jester@phys.soton.ac.uk.

${ }^{10}$ We use a flat, accelerating cosmology with $H_{0}=71 \mathrm{~km} \mathrm{~s}^{-1} \mathrm{Mpc}^{-1}, \Omega_{m}=$ 0.27 , and $\Omega_{\Lambda}=0.73$.
}

ray tube ${ }^{11}$ may help constrain such problems as how the energy of a jet is dissipated and the jet decelerated (e.g., Georganopoulos \& Kazanas 2003, 2004; Tavecchio et al. 2006) or how the radio lobes relate to the X-ray jet. Details of the structure of the narrow jet (Schwartz et al. 2006b) and of our Hubble Space Telescope observations of the system will be presented elsewhere.

\section{IMAGES OF PKS $1055+201$}

Chandra observation obs/05733 yielded a 31.595 ks livetime observation of PKS $1055+201$. Figure 1 presents radio and X-ray images of the system PKS $1055+201$. The radio jet to the north closely resembles the X-ray jet up to the terminal $\mathrm{X}$-ray hot spot about $21^{\prime \prime}$ from the quasar. That hot spot is associated with the eastern peak of a bright, resolved radio hot spot. A radio extension to the north of this hot spot is not reproduced in the X-ray image. Both the radio and X-ray jet start out at a position angle within a few degrees of north, close to the VLBA jet direction of $-5^{\circ}$ reported by Kellerman et al. (2004). They reported superluminal motion with an apparent velocity $v=(10 \pm 4) c$, for a component 3 mas from the core.

Binning the X-ray data $(0.5-7 \mathrm{keV})$ into 1 " bins shows enhanced X-ray emission from a region about $16^{\prime \prime}$ wide and parallel to the radio and X-ray jet to the north (Fig. 2). A roughly symmetric region of enhanced X-rays also appears to the south. Its position suggests that it surrounds the unseen counterjet, which powers the southern radio lobe.

We quantify the extended X-ray emission by considering the profiles in Figure 3. The left panel gives the profiles across the corresponding regions shown in the right panel. The X-ray counts are the sum of the data projected into bins 1 " wide along the solid lines in the right panel. The abscissae are arbitrarily offset to line up the centroids of the emission in each case. The dashed line gives the background rate expected, 1.31 counts per bin, based on the region marked "B." The red line gives the profile across the ACIS readout streak from the bright quasar core. This represents the undersampled profile of an unresolved line source. The actual counts in the streak profile are multiplied by 4.2 to give the number of counts that the narrow jet is expected to contribute to the profile (green his-

\footnotetext{
${ }^{11}$ We use "tube" rather than "lobe" or "cocoon" to avoid prejudging the nature of this region.
} 


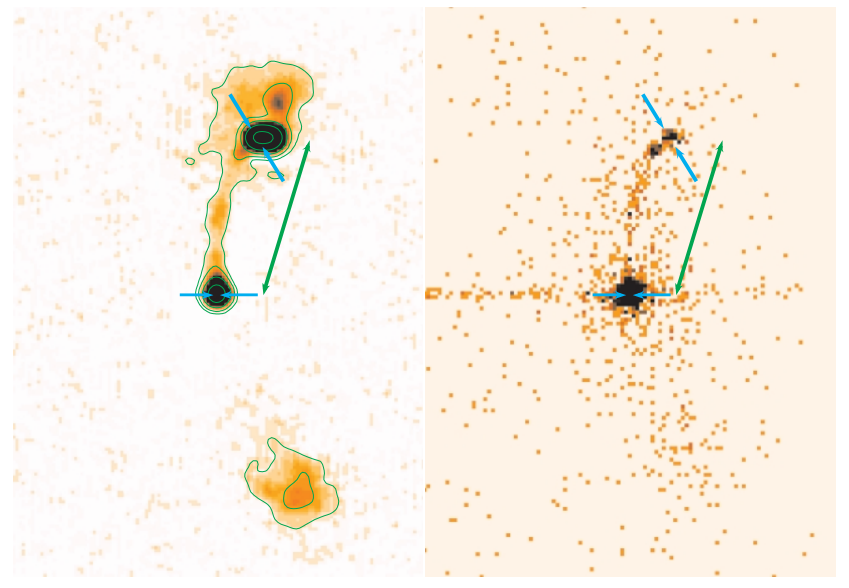

FIG. 1.-X-ray and radio images of PKS $1055+201$. Right panel plots the $0.5-7 \mathrm{keV}$ X-ray data, in 0"49 bins. Left panel shows a $1.46 \mathrm{GHz}$ VLA Aarray image, restored with a 1 ".65 61 1.50 beam. Both images are to the same scale, with the $21^{\prime \prime}$-long arrow indicating the length of the X-ray jet. Radio contours are factors of 4 starting from $1.5 \mathrm{mJy}^{-1}$ beam $^{-1}$, with a peak of 768 $\mathrm{mJy}_{\text {beam }}{ }^{-1}$. The rms noise is $0.6 \mathrm{mJy}_{\text {beam }}^{-1}$. The quasar and terminal Xray hot spot are marked with pairs of arrows. The horizontal streak through the quasar in the X-ray image is the ACIS readout artifact.

togram) from the north region. ${ }^{12}$ The south region profile is given by the blue histogram. Errors on the tube region profiles are the square root of the number of counts in the bin. The dotted curve is the profile predicted for a uniformly emitting cylinder of diameter $16^{\prime \prime}$.

${ }^{12}$ Since the jet curves a small amount, its contribution to the profile is slightly broader than the line response function given by the readout streak.
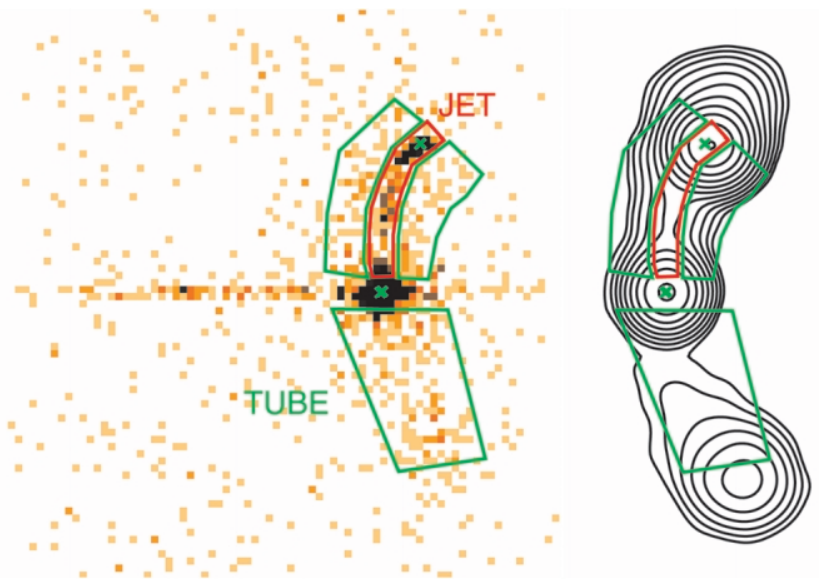

FIG. 2.-Left: X-rays plotted in 1" bins. The narrow X-ray jet is denoted by the region enclosed in red. The extended X-ray "tube" of emission is indicated by the three regions enclosed in green. Right panel shows $1.425 \mathrm{GHz}$ VLA-B contours, which are factors of $2 \times 1.2 \mathrm{mJy}^{-1}$ beam $^{-1}$, with a $1.54 \mathrm{Jy}_{\text {beam }}{ }^{-1}$ peak. The rms noise is $0.4 \mathrm{mJy}$ beam $^{-1}$. The south tube region flux is about $12 \mathrm{mJy}$, and the north region is consistent with a similar flux plus the narrow jet smeared by the 5 " beam FWHM. Scales are the same in both panels, as are the "tube" and "jet" regions. The same points indicated in Fig. 1 are here marked with an $\mathrm{X}$.

We can make some simple estimates by modeling the extended X-ray brightness as from a uniformly emitting cylinder. We consider the observed energy range $0.5-7 \mathrm{keV}$ and subtract 0.0685 counts $\operatorname{arcsec}^{-2}$ background to derive all the numbers quoted. From the measured counts in the regions outside the jet (Fig. 2, green areas) we estimate that 47.5 of the 243.8 background-subtracted counts in the jet region (Fig. 2, red
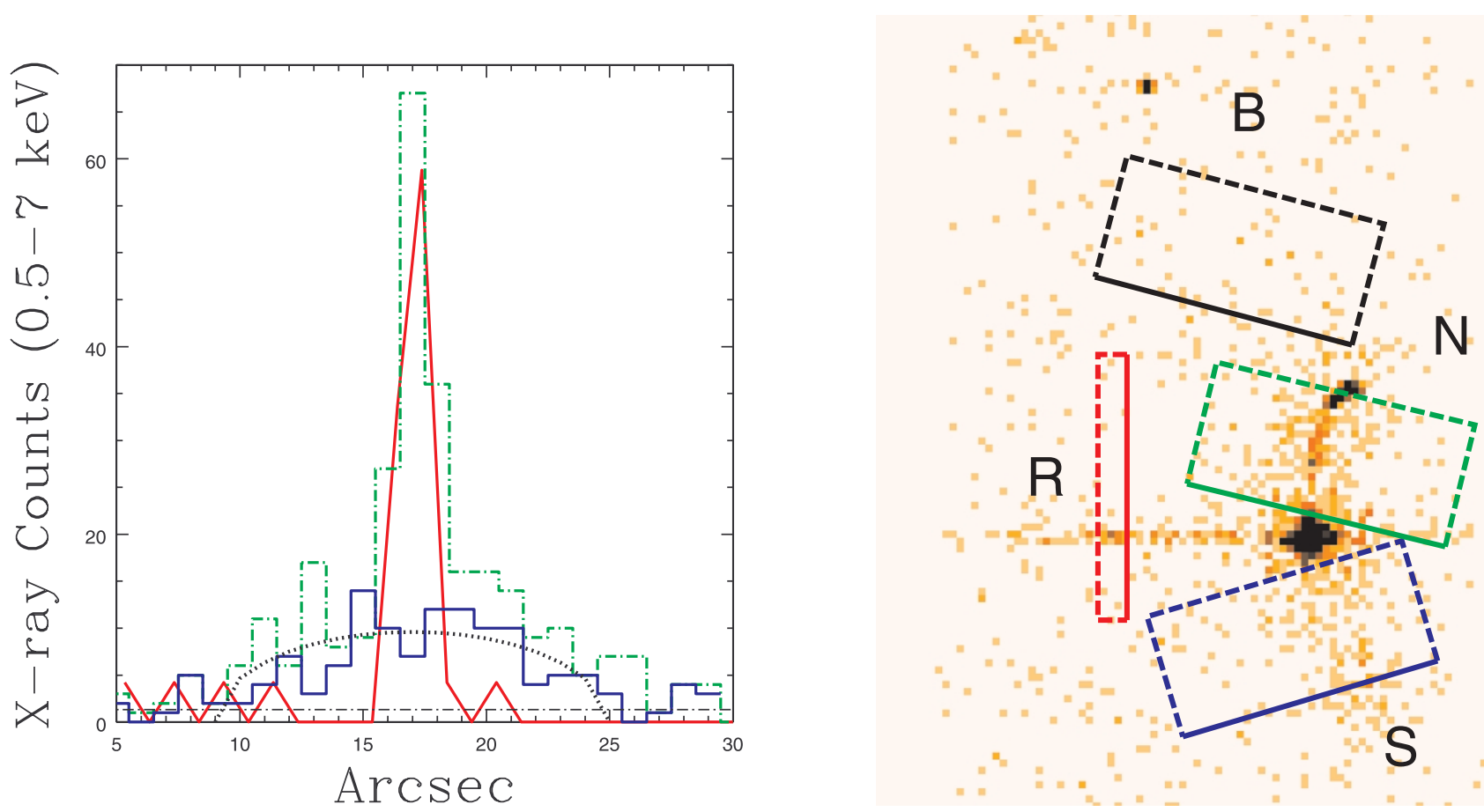

FIG. 3.-Left panel shows the profiles of projections across the regions defined in the X-ray image in the right panel. Green dot-dashed histogram shows the northern jet and tube ("N"), blue solid histogram shows the southern tube ("S"), the dashed line gives the background as determined from region "B," and the red line gives the profile across the readout streak ("R"). The heavy dotted line models the extended X-ray profiles as from a uniform cylinder. The projection collapses counts within the dashed rectangles into 1" bins along each solid line. We plot from left to right along that line of projection, offsetting the profiles so that the centroids are aligned. 
areas) are due to projection of the extended tube region in front and behind the jet. We then infer that there are $196.3 \pm 14.1$ counts intrinsic to the jet, and $175.6 \pm 14.0$ and $145.2 \pm 13.7$ counts in the north and south tube regions, respectively. Using the spectral models described in the next section, we estimate rest-frame $2-10 \mathrm{keV}$ luminosities of approximately $1.8 \times 10^{44}$ ergs $\mathrm{s}^{-1}$ for the jet, and $1.8 \times 10^{44}$ and $1.5 \times 10^{44} \mathrm{ergs} \mathrm{s}^{-1}$ for the north and south tube regions, respectively.

\section{X-RAY SPECTRA}

We use XSPEC (Arnaud 1996) to fit the X-ray spectra of the extended X-ray tube emission and of the jet, where those two regions are defined in Figure 2. In all cases we fix the Galactic absorption to column density $N_{\mathrm{H}}=1.9 \times 10^{20} \mathrm{~cm}^{-2}$ (Stark et al. 1992). We take the energy regions $0.5-5 \mathrm{keV}$ (since there are few counts between 5 and $7 \mathrm{keV}$ ), bin the data into 25 counts or more, and use a $\chi^{2}$ goodness-of-fit estimator. We first fitted the spectrum of the background to a power law. Then we fitted the spectrum of the extended X-ray emission to the sum of the normalized, fixed-background fit plus either an absorbed power law or an absorbed thermal (MEKAL) model. Then we fitted the spectrum of the jet to the sum of the normalized, fixed-background fit plus the normalized, fixed fit to the extended region for each of the two cases, plus an absorbed power law. We quote $90 \%$ confidence error regions for one interesting parameter, either the temperature or power-law photon index $\Gamma$.

The background is fitted by a power law $\Gamma=0.54 \pm 0.23$. Background contributes an estimated $12 \%$ of the extendedregion spectrum and $2 \%$ of the jet-region spectrum. The MEKAL fit to the extended region gives $k T=8.6_{-4.5}^{+34} \mathrm{keV}$ with a $\chi^{2}$ of 14.05 for 8 degrees of freedom, where we have fixed the abundances at cosmic values. The power-law fit for this region gives $\Gamma=1.73 \pm 0.33$ with a $\chi^{2}$ of 9.4 . The power law is clearly a better fit to the extended region, with a probability $P\left(>\chi^{2}\right)=0.31$, but the thermal fit cannot be rejected since $P\left(>\chi^{2}\right)=0.08$, and it is interesting to interpret both models. The jet power-law index is $\Gamma=2.15 \pm 0.33$ with $\chi^{2}$ of 2.33 for 6 degrees of freedom if the MEKAL fit is assumed for the extended region, and $\Gamma=2.11 \pm 0.33$ with $\chi^{2}$ of 2.38 if the power-law fit is assumed. The spectral shape deduced for the jet is not sensitive to the model of the extended region, and we shall consider only the parameters obtained from assuming the power-law fit to the extended emission. Such a fit for the jet region has $P\left(>\chi^{2}\right)=0.88$.

The $\Gamma$ values for the jet and tube regions are consistent at the $90 \%$ confidence level. A joint absorbed power-law fit of the extended and jet regions, forcing the photon index to be the same in both, results in an acceptable $\chi^{2}$ of 13.81 for 15 degrees of freedom, with $\Gamma=1.92 \pm 0.19$, to $90 \%$ confidence.

\section{DISCUSSION}

We model the jet assuming minimum energy conditions between the magnetic field and relativistic particles, with equal electron and proton energy densities. We assume the X-ray emission is inverse Compton (IC) scattering from the cosmic microwave background (CMB), which requires that the jet be relativistically beamed near to our line of sight (Tavecchio et al. 2000; Celotti et al. 2001). Details of these assumptions are the same as in Schwartz et al. (2006a). We derive mean jet parameters (Schwartz et al. 2006b) that are roughly as follows: magnetic field in the rest frame $B \approx 10 \mu \mathrm{G}(1 \mathrm{nT}=10 \mu \mathrm{G})$; electron density in the rest frame $n_{e} \approx 2 \times 10^{-8} \mathrm{~cm}^{-3}$ (between
Lorentz factors $\gamma$ from 80 to $10^{5}$ and with an assumed radio energy index of $\alpha=0.7$ ); Doppler factor $\delta \approx 6$; kinetic flux $\approx 2 \times 10^{45} \mathrm{ergs} \mathrm{s}^{-1}$; angle to the line of sight $\theta \leq 9^{\circ}$; and deprojected length $\approx 1 \mathrm{Mpc}$ when we assume the maximum $\theta=$ $9^{\circ}$. These quantities become $B \approx 60 \mu \mathrm{G}, n_{e} \approx 10^{-6} \mathrm{~cm}^{-3}$, and $\delta \approx 4.5$ at the terminal $\mathrm{X}$-ray hot spot.

We consider two mechanisms for the extended X-ray emission: thermal bremsstrahlung from gas heated by the jet or IC/ $\mathrm{CMB}$ from relativistic electrons whose ultimate energy source is from the jet. We consider the total volume $V$ of two cylinders, each $16^{\prime \prime}=130 \mathrm{kpc}$ in diameter by $1 \mathrm{Mpc}$ in length, and emitting a total $3.3 \times 10^{44} \mathrm{ergs} \mathrm{s}^{-1}$ in the rest-frame $2-10 \mathrm{keV}$ band. We assume that the cylinder is not moving relativistically with respect to the $\mathrm{CMB}$, since the two sides of the source are of similar length, and that the X-ray emissivity is apparently uniform throughout the cylinder volumes.

If the extended X-ray emission is thermal and uniform, then $k T=8.6 \mathrm{keV}$ and $n_{e}=0.004 \mathrm{~cm}^{-3}$. The cooling time would be $1.2 \times 10^{10} \mathrm{yr}$, much longer than the age of the universe at $z=1.1, t=5.5 \times 10^{9}$ yr. The total energy would be $E=$ $3 n_{e} k T V \approx 1.6 \times 10^{62} \mathrm{ergs}$. Assuming that there is a counterjet of similar power, the jets supply a kinetic flux of $4 \times 10^{45} \mathrm{ergs}$ $\mathrm{s}^{-1}$, requiring $1.3 \times 10^{9}$ yr to supply the total energy. A duty cycle of $25 \%$ seems high but could be reduced if the gas is nonuniform. Then the $\left\langle n_{e}\right\rangle$ and $E$ could be $\sim 10$ times smaller while maintaining $\left\langle n_{e}^{2}\right\rangle=(0.0043)^{2} \mathrm{~cm}^{-6}$ in order to produce the observed X-ray luminosity. Alternatively, one might infer that previous outbursts of the jet averaged a kinetic flux that was a factor of several more luminous than the jet we currently observe, or that the present jet has a ratio of proton to electron energy a factor of several higher than the value unity that we assume. The confinement of such a gas is problematical in the absence of a rich cluster medium, for whose presence we have no indication. Nonetheless, we could be observing a system similar to Cyg A (Wilson et al. 2006), but tilted closer to our line of sight and substantially larger. Measurement of the rotation measure might test for the presence of a thermal tube, or of a hot cluster medium. If magnetic fields of the order of $0.1 \mu \mathrm{G}$ were present in such an extended medium, we would expect a rotation measure $\mathrm{RM} \approx 20 \times\left(B_{\|} / 0.1 \mu \mathrm{G}\right)$.

In the case of nonthermal emission of X-rays from the extended region, the low level of $1.4 \mathrm{GHz}$ radio emission implies that the magnetic field is much lower than $14 \mu \mathrm{G}$, which is the field that would have the same energy density as the CMB at the redshift $z=1.11$. With an upper limit of $10 \mathrm{mJy}$ for the radio emission, the equipartition field would be about $6 \mu \mathrm{G}$. We will assume the $\mathrm{X}$-ray emission is IC/CMB from a region not in bulk relativistic motion with respect to the microwave background. Under those conditions, we deduce a density of electrons with Lorentz factor above $\gamma=80$ of $2.2 \times 10^{-8} \mathrm{~cm}^{-3}$, comparable to the density within most of the length of the jet. The electron lifetimes are limited by scattering on the CMB, and are of order $10^{8} \mathrm{yr}$ for electrons producing inverse Compton radiation at $1 \mathrm{keV}$, but less than $10^{7} \mathrm{yr}$ for the higher energy electrons emitting $1.46 \mathrm{GHz}$ synchrotron radiation.

The nonthermal scenario is consistent with an interpretation wherein the jet is generating X-ray-emitting lobes throughout a lifetime of several times $10^{7} \mathrm{yr}$, and these lobes are decelerated to substantially less than the speed of light, while the jet continues to propagate with a large Lorentz factor. To diffuse laterally to the observed radial extent of $65 \mathrm{kpc}$ in the $10^{8} \mathrm{yr}$ lifetime of the X-ray-emitting electrons implies a transverse velocity of order $600 \mathrm{~km} \mathrm{~s}^{-1}$. While radio lobes are ubiquitous, and while X-ray emission has been detected from radio lobes 
(e.g., Croston et al. 2005 and references therein), the present observation of PKS $1055+201$ is unique in that both a prominent X-ray jet and a surrounding X-ray tube region are seen, and in that the latter does not correlate morphologically with the weak, extended radio emission at $1.46 \mathrm{GHz}$.

This work was supported by NASA contract NAS8-39073 to the Chandra X-Ray Center and SAO SV1-61010 to MIT, and NASA grant GO2-3151C to SAO. J. M. G. was supported by NASA grants GO4-5124X and GO5-6116A. E. S. P. acknowledges support from NASA LTSA grant NAG5-9997.
S. J. was supported by the MPI für Astronomie through an Otto Hahn fellowship. Part of this research was performed at the Jet Propulsion Laboratory, California Institute of Technology, under contract to NASA. We thank M. Hardcastle and D. Harris for discussions and comments. This research used the NASA Astrophysics Data System Bibliographic Services and the NASA/IPAC Extragalactic Database (NED), which is operated by the Jet Propulsion Laboratory, California Institute of Technology, under contract with the National Aeronautics and Space Administration.

\section{REFERENCES}

Arnaud, K. A. 1996, in ASP Conf. Ser. 101, Astronomical Data Analysis Software and Systems V, ed. G. H. Jacoby \& J. Barnes (San Francisco: ASP), 17

Bolton, J. G., Kinman, T. D., \& Wall, J. V. 1968, ApJ, 154, L105

Celotti, A., Ghisellini, G., \& Chiaberge, M. 2001, MNRAS, 321, L1

Chartas, G., et al. 2000, ApJ, 542, 655

Croston, J. H., Hardcastle, M. J., Harris, D. E., Belsole, E., Birkinshaw, M., \& Worrall, D. M. 2005, ApJ, 626, 733

Gelbord, J., et al. 2005, in X-Ray and Radio Connections, ed. L. O. Sjouwerman \& K. K. Dyer (Socorro: NRAO), http://www.aoc.nrao.edu/events/xraydio Georganopoulos, M., \& Kazanas, D. 2003, ApJ, 589, L5

- 2004, ApJ, 604, L81

Kellerman, K. I., et al. 2004, ApJ, 609, 539
Marshall, H. L., et al. 2005, ApJS, 156, 13

Pilkington, J. D. H., \& Scott, J. F. 1965, MmRAS, 69, 183

Schwartz, D. A., et al. 2000, ApJ, 540, L69

- 2006a, ApJ, 640, 592

2006b, in Proc. X-Ray Universe 2005, ed. A. Wilson (ESA SP-604; Noordwijk: ESA), 579

Shimmins, A. J., \& Day, G. A. 1968, Australian J. Phys., 21, 377

Stark, A. A., et al. 1992, ApJS, 79, 77

Tavecchio, F., Maraschi, L., Sambruna, R. M., Gliozzi, M., Cheung, C. C., Wardle, J. F. C., \& Urry, C. M. 2006, ApJ, 641, 732

Tavecchio, F., Maraschi, L., Sambruna, R. M., \& Urry, C. M. 2000, ApJ, 544, L23

Wilson, A. S., Smith, D. A., \& Young, A. J. 2006, ApJ, 644, L9 